\title{
Increased Dementia and Mild Cognitive Impairment (MCI) by Impaired Glucose Metabolism
}

\author{
Hiroshi Bando ${ }^{1,2 *}$ \\ ${ }^{1}$ Tokushima University / Medical Research, Japan \\ ${ }^{2} J a p a n$ Low Carbohydrate Diet Promotion Association (JLCDPA), Japan \\ *Corresponding author: Hiroshi BANDO, Tokushima University /Medical Research, Nakashowa 1-61, Tokushima 770-0943, Japan. \\ To Cite This Article: Hiroshi BANDO. Increased Dementia and Mild Cognitive Impairment (MCI) by Impaired Glucose Metabolism. Am J Biomed \\ Sci \& Res. 2019 - 2(5). AJBSR.MS.ID.000604. DOI: 10.34297/AJBSR.2019.02.000604
}

Received: April 18, 2019 | Published: April 29, 2019

\begin{abstract}
Keywords: Alzheimer's disease; Mild cognitive impairment; Low carbohydrate diet; Population attributable fraction; Dietary approaches to stop
\end{abstract} hypertension

Abbrevations: AD: Alzheimer's Disease; MCI: Mild Cognitive Impairment; LCD: Low Carbohydrate Diet; T2DM: Type 2 Diabetes Mellitus; PAF: Population Attributable Fraction; DASH: Dietary Approaches to Stop Hypertension

\section{Introduction}

Diabetes has been evaluated as a key risk factor for mild cognitive impairment (MCI) and dementia [1-3]. Then, increasing the prevalence of diabetes and prediabetes would contribute the burden of MCI and dementia in the healthcare systems in each country [4]. Consequently, the links between diabetes, MCI and dementia would be investigated for the improvement of these diseases in the future [5].

A metabolic state that exists between normal glucose variability and type 2 diabetes mellitus (T2DM) would be defined as prediabetes. Recently the ratio of the prediabetes in adult has been rapidly increasing, which would be estimated as about $35 \%$ in the USA and UK, and up to about 50\% in China [6].

Across the world, approximately 47 million people were suffering from dementia in 2015 , and this number is estimated to triple by 2050 [2]. Furthermore, the global cost of dementia in 2015 was probably to be US $\$ 818$ billion, and this figure will be increased as the subjects with dementia will rise [2].

There have been several statistic data about dementia and diabetes. T2DM patients for 5 years or more tend to show increased dementia risk of $40-60 \%$ [7]. The risk for dementia seems to be increased for higher mean blood glucose in the patients less than 84 years old. The treatment of not insulin, but oral hypoglycemic agents would lead to lower risk of developing dementia [7]. Furthermore, hypoglycaemia seems to show a bi-directional association with cognitive impairment [8].

T2DM has been evaluated to increase the risk for cognitive dysfunction across all cognitive domains. The relative risks of developing dementia and vascular dementia in people with T2DM would be 1.51 and 2.48 , respectively [9].

Increased dementia risk in general people was believed to be associated with elevated blood cholesterol values. For T2DM patients, however, there are discrepancy of the ratio for dyslipidemia in the case of both of increased or decreased risks of cognitive impairment [10]. After that, cholesterol values do not influence the risk of progression from MCI to dementia [1].

Certain relationship exists between glucose variability and Alzheimer's disease (AD). When the patient has already established $\mathrm{AD}$, the level of cognitive impairment has been correlated with the reduced level of glucose uptake. Moreover, some evidence was found that changed energy metabolism in the brain would induce the development to the level of $\mathrm{AD}[11,12]$.

There are three kinds of ketone bodies (KB), which are produced from fat metabolism in the body. Among them, 3-OHBA (3-hydroxybutyric acid) and AcAc (acetoacetic acid) have the function of KB. They can be used for production of the energy, and the energy is consumed in brain and muscle.

From pathophysiological mechanism of $\mathrm{AD}$, various approach to the nutrition therapy for $\mathrm{AD}$ would become crucial problems in the future. The reason is that AD has been associated with impaired glucose metabolism from various research so far [11]. Further, insulin resistance and impaired insulin secretion would be also significant problem for AD [13].

There have been many kinds of nutritional therapy introduced so far. Among them, they can be generally divided into two categories, 
which are low carbohydrate diet (LCD) and calorie restriction diet (CRD) in the diabetic medical practice. On the other hand, in the field of management of dementia and MCI, the recommended and rather popular regimens would be the Dietary Approaches to Stop Hypertension (DASH) and Mediterranean-DASH Intervention for Neurode generative Delay (MIND) diets [14].

For the prevention of $\mathrm{AD}$, dietary recommendation would be the similar to that of diabetes, and diseases of metabolic syndrome [15]. Among them, Mediterranean diet would be beneficial type of nutrition therapy because of its lower risk rate of AD.

There are several modifiable risk factors for dementia. For the study on dementia risk, midlife life would be 45-65 years old, and later life would be older than 65 years [2]. The research was focused on estimating the population attributable fraction (PAF). It is the reduction ratio in new cases for a certain period if one risk factor was completely distinguished. There have been several related factors such as diabetes, hypertension, obesity, physical inactivity, smoking depression and low educational attainment [16]. Potentially modifiable risk factors for dementia in later life more than 65 years old was calculated. Those data of relative risk were that smoking 1.6, depression 1.9, physical inactivity 1.4 , social isolation 1.6, and diabetes 1.5. Among them, total weighted PAF adjusted for communality was 35\% [2].

Furthermore, a systematic review and meta-analysis for social isolation and incident dementia were applied to calculate its PAF [17]. It included social contact (telephone or face-to-face contact), social participation (participation in community activities) and loneliness (feeling at dissatisfaction from social contact). The value of weighted relative ratio (RR) for incident dementia from less frequency of social contact was 1.57 [17].

In recent years, depression is one of the important problems. Patients with T2DM has been estimated to have depression up to $39 \%$ [18]. Further, those people with T2DM and depression will have twice as likely to become dementia.

On the other hand, diabetic patients have been increasing so far and estimated to be approximately 700 million by 2045 [19]. Several controversies have been found about the diet therapy such as LCD and CRD through various clinical research for long years. There have been some guidelines from American Diabetes Association (ADA) and American College of Physicians (ACP) in recent years, in which the evaluation and management for $\mathrm{HbA1c}$ and the goal have some difference [20].

For diabetic therapy, LCD has been applied and known in European countries so far. On the other hand, LCD has been initiated by authors and co-researchers and developed through lectures and books [21]. There are three useful kinds of LCD including petite, standard and super [22]. Further, we have reported clinical significance of role for Ketone Bodies (KB) in the axis of fetusplacenta-newborn-mother and in the situation of clinical LCD continuation [23].

Summarized mentioned above, diabetes has been risking factor of dementia and MCI. These diseases have been increased and become medical and social problem across the world. This article would be a reference for future research and clinical practice.

\section{References}

1. Cooper C, Sommerlad A, Lyketsos CG, Livingston G (2015) Modifiable predictors of dementia in mild cognitive impairment: a systematic review and meta-analysis. Am J Psychiatry 172(4): 323-334.

2. Livingston G, Sommerlad A, Orgeta V, Costafreda SG, Huntley J, et al. (2017) Dementia prevention, intervention, and care. Lancet 390(10113): 2673-2734.

3. Davis WA, Zilkens RR, Starkstein SE (2017) Dementia onset, incidence and risk in type 2 diabetes: a matched cohort study with the Fremantle Diabetes Study Phase I. Diabetologia 60(1): 89-97

4. Cho NH, Shaw JE, Karuranga S, Huang Y, da Rocha Fernandes JD, et al. (2018) IDF Diabetes Atlas: global estimates of diabetes prevalence for 2017 and projections for 2045. Diabetes Res Clin Pract 138: 271-281.

5. Biessels GJ, Strachan MWJ, Visseren FLJ (2014) Dementia and cognitive decline in type 2 diabetes and prediabetic stages: towards targeted interventions. Lancet Diabetes Endocrinol 2(3): 246-255.

6. Mainous AG, Tanner RJ, Baker R, Cilia E Zayas, Christopher A Harle, et al. (2014) Prevalence of prediabetes in England from 2003 to 2011: population-based, cross-sectional study. BMJ Open 4(6): e005002.

7. Parikh Niraj M, Morgan Robert O, Kunik Mark E, Schulz PE, Johnson ML, et al. (2011) Risk factors for dementia in patients over 65 with diabetes. Int J Geriatr Psychiatry 26(7): 749-757.

8. Yaffe K, Falvey CM, Hamilton N, Harris TB, Simonsick EM et al (2013) Association between hypoglycemia and dementia in a biracial cohort of older adults with diabetes mellitus. JAMA Intern Med 173(14): 13001306.

9. Cheng G, Huang C, Deng H, Wang H (2012) Diabetes as a risk factor for dementia and mild cognitive impairment: a meta-analysis of longitudinal studies. Intern Med J 42(5): 484-491.

10. Umegaki H, Satoshi S, Shinozaki T, Araki A, Sakurai T, et al. (2012) Risk factors associated with cognitive decline in the elderly with type 2 diabetes: pooled logistic analysis of a 6-year observation in the Japanese elderly diabetes intervention trial. Geriatr Gerontol Int 12(1): 110-116.

11. An Y, Varma VR, Varma S, Casanova R, Dammer E, Plet-nikova O, et al. (2018) Evidence for brain glucose dys-regulation in Alzheimer's disease. Alzheimers Dement 14(3): 318-329.

12. Abolhassani N, Leon J, Sheng ZJ, Oka S, Hamasaki H, Iwaki T, et al. (2017) Molecular pathophysiology of impaired glucose metabolism, mitochondrial dysfunc- tion, and oxidative DNA damage in Alzheimer's disease brain. Mech Ageing Dev 161: 95-104.

13. Reger MA, Watson GS, Green PS, Wilkinson CW, Baker LD, Cholerton B, et al. (2008) Intranasal insulin improves cognition and modulates betaamyloid in early AD. Neurology 70(6): 440-448.

14. Levitan EB, Lewis CE, Tinker LF, Eaton CB, Ahmed A, Manson JE, et al. (2013) Mediterranean and DASH diet scores and mortality in women with heart fail ure: The Women's Health Initiative. Circ Heart Fail 6(6): 1116-1123.

15. Creegan R, Hunt W, McManus A, Rainey-Smith SR (2015) Diet, nutrients and metabolism: Cogs in the wheel driving Alzheimer's disease pathology? Br J Nutr 113(10): 1499-1517.

16. Norton S, Matthews FE, Barnes DE, Yaffe K, Brayne C (2014) Potential for primary prevention of Alzheimer's disease: an analysis of populationbased data. Lancet Neurol 13: 788-794.

17. Kuiper JS, Zuidersma M, Oude Voshaar RC, Stolk RP, Smidt N, et al. (2015) Social relationships and risk of dementia: a systematic review and metaanalysis of longitudinal cohort studies. Ageing Res Rev 22: 39-57.

18. Perrin NE, Davies MJ, Robertson N, Snoek FJ, Khunti K, et al. (2017) The prevalence of diabetes-specific emotional distress in people with type 
2 diabetes: a systematic review and meta-analysis. Diabet Med 34(11): 1508-1520.

19. Ogurtsova K, da Rocha Fernandes JD, Huang Y, Linnenkamp U, Guariguata L, Cho NH, et al. (2017) IDF Diabetes Atlas: global estimates for the prevalence of diabetes for 2015 and 2040. Diabetes Res Clin Pract 128 40-50.

20. American Diabetes Association (2018) Pharmacologic Approaches to Glycemic Treatment: Standards of Medical Care in Diabetes-2018. Diabetes Care 41(1): S73-S85.
21. Ebe K, Bando H, Yamamoto K, Bando M, Yonei Y (2018) Daily carbohydrate intake correlates with $\mathrm{HbA1c}$ in low carbohydrate diet (LCD). J Diabetol 1(1): 4-9.

22. Bando H, Ebe K, Muneta T, Bando M, Yonei Y (2017) Clinical Effect of Low Carbohydrate Diet (LCD): Case Report. Diabetes Case Rep 2: 124.

23. Muneta T, Kawaguchi E, Hayashi M, Bando H, Ebe K (2019) Normalized glucose variability by Low Carbohydrate Diet (LCD) in CGM study. Asp Biomed Clin Case Rep 2(1): 22-27. 